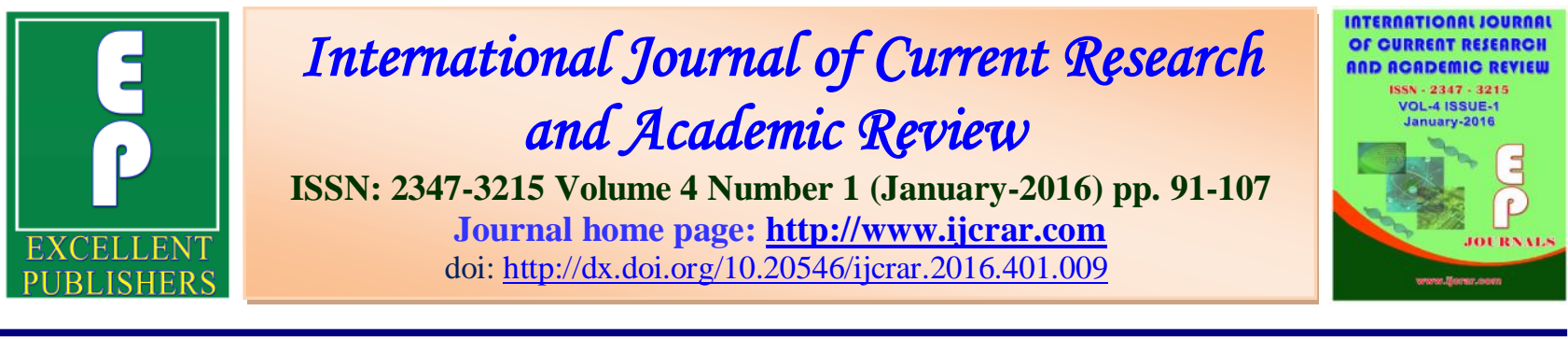

\title{
MHD flow of heat and mass transfer over an unsteady stretching sheet in a micropolar fluid with heat source/sink and chemical reaction
}

\author{
M. Sreedhar Babu ${ }^{*}$, M.Lavanya ${ }^{2}$ and G. Venkata Ramanaiah ${ }^{2}$ \\ ${ }^{1}$ Department of Applied Mathematics, Y.V.University, Kadapa Andhra Pradesh, India \\ ${ }^{2}$ Research scholar, Dept. of Applied Mathematics, Y.V.University, Kadapa, \\ Andhra Pradesh, India
}

*Corresponding author

\begin{tabular}{|c|c|}
\hline KEYWORDS & A B S T R A C T \\
\hline $\begin{array}{l}\text { Unsteady flow, } \\
\text { Heat and Mass } \\
\text { Transfer, } \\
\text { Micropolar fluid, } \\
\text { MHD, } \\
\text { Stretching sheet, } \\
\text { Heat source or Sink, } \\
\text { Chemical reaction. }\end{array}$ & $\begin{array}{l}\text { The effect of heat source/sink on magnetohydrodynamic (MHD) laminar flow } \\
\text { of an incompressible micropolar fluid over an unsteady stretching sheet with } \\
\text { chemical reaction is investigated. Using the similarity transformations, the } \\
\text { governing equations have been transformed into a system of ordinary } \\
\text { differential equations. These differential equations are highly nonlinear which } \\
\text { cannot be solved analytically. Therefore, fourth order Runge-Kutta method } \\
\text { along with shooting technique has been used for solving it. Numerical results } \\
\text { are obtained for the skin-friction coefficient, the local Nusselt number and } \\
\text { concentration as well as the velocity, temperature and concentration profiles } \\
\text { for different values of the governing parameters, namely, unsteadiness } \\
\text { parameter, material parameter, magnetic parameter, Prandtl number, heat } \\
\text { source/sink parameter, Schmidt number and chemical reaction parameter. }\end{array}$ \\
\hline
\end{tabular}

\section{Introduction}

In recent years, the dynamics of micropolar fluids, originating from the theory of Eringen (1), has been a popular area of research. This theory takes into account the effect of local rotary inertia and couple stresses arising from practical microrotation action. This theory is applied to suspensions, liquid crystals, polymeric fluids and turbulence. This behavior is familiar in many engineering and physical applications. Also, the study of boundary layer flows of micropolar fluids over a stretching surface has received much attention because of their extensive applications in the field of metallurgy and chemical engineering for example, in the extrusion of polymer sheet from a die or in the drawing of plastic films. $\mathrm{Na}$ and Pop (2) investigated the boundary layer flow of a micropolar fluid past a stretching wall. Desseaux and Kelson (3) studied the flow of a micropolar fluid bounded by a stretching sheet. Takhar et al (4) studied the mixed convection flow of a micropolar fluid over a stretching sheet. 
Elbashbeshy (5) investigated the heat transfer over a stretching surface with variable surface heat flux. Recently, Bachok et al. (6) studied the flow and heat transfer over an unsteady stretching sheet in a micropolar fluid with prescribed surface heat flux.

Some specific industrial applications such as in polymer processing technology, which involve cooling of continuous strip or filaments, these elements, are drawn through a quiescent fluid. During the process, strips are sometimes stretched. The properties of the final product depend on the rate of cooling. Thus, rate of cooling can be greatly controlled by the use of electrically conducting fluid and the application of the magnetic field. Another use of application of magnetic field is found in the purification process of molten metal's from non-metallic inclusions.

Numerous attempts have been made to analysis the effect of transverse magnetic field on boundary layer flow heat and mass transfer characteristics of electrically conducting fluid. Vajravelu and Rollins (7) studied heat transfer in an electrically conducting fluid over a stretching surface by taking into account the magnetic field only.

Prasad et al. (8) examined the influence of variable fluid properties on the hydromagnetic flow and heat transfer over a nonlinearly stretching sheet. In all these works effect of electric field has been neglected which is also one of the important parameters to alter the momentum and heat transfer characteristics in a Newtonian boundary layer flow. The problems of coupled heat and mass transfer in MHD two-dimensional flow, the effects of Ohmic heating have not been studied by previous authors. However, it is more realistic to include this effect to explore the impact of the magnetic field on the thermal transport in the boundary layer. Haque et al.(9) studied micropolar fluid behavior on steady magneto hydrodynamics free convection flow and mass transfer through a porous medium with heat and mass fluxes. Rebhi(10) studied unsteady natural convection heat and mass transfer of micropolar fluid over a vertical surface with constant heat flux. Olajuwon and Oahimire (11) studied the unsteady free convection heat and mass transfer in a MHD micropolar fluid in the presence of thermo diffusion and thermal radiation.

In several physical problems such as fluids undergoing exothermic or endothermic chemical reactions, it is important to study the effects of heat generation and absorption. The presence of heat generation or absorption may alter the temperature distribution in the fluid which in turn affects the particle deposition rate in systems such as nuclear reactors, electronic chips, and semiconductor wafers. The exact modeling of internal heat generation or absorption is difficult but some simple mathematical models may express its average behavior for most physical situations. Cortell (12) studied the flow and heat-transfer in a porous medium over a stretching surface with internal heat generation or absorption. Heat generation/ absorption and viscous dissipation effects on MHD flow of a micropolar fluid over a moving permeable surface embedded in a non-Darcian porous medium has been studied by Mahmoud (13). Mostafa et al. (14) analyzes the MHD flow and heat transfer of a micropolar fluid over a stretching surface with heat generation (absorption) and slip velocity. Khedr et al.(15) studied MHD flow of a micropolar fluid past a stretched permeable surface with heat generation or absorption. Abo-Eldahab and Abd El-Aziz (16) studied MHD threedimensional flow over a stretching sheet in a non-Darcian heat generation or absorption effects. 
Damseh et al. (17) investigate the combined heat and mass transfer by natural convection of a micropolar, viscous and heat generating or absorbing fluid flow near a continuously moving vertical permeable infinitely long surface in the presence of a first-order chemical reaction. Kandasamy et al.(18) studied the nonlinear MHD flow, with heat and mass transfer characteristics, of an incompressible, viscous, electrically conducting, Boussinesq fluid on a vertical stretching surface with chemical reaction and thermal stratification effects. Seddek(19) studied the effects of chemical reaction, thermophoresis and variable viscosity on steady hydromagnetic flow with heat and mass transfer over a flat plate in the presence of heat generation/absorption. Patil and Kulkarni(20) studied the effects of chemical reaction flow of a polar fluid through porous medium in the presence of internal heat generation.

The present study contains an analysis of the effects of magnetohydrodynamic laminar flow of a micropolar fluid over an unsteady stretching sheet by taking heat source/sink, mass transfer and chemical reaction into account. Using the similarity transformations, the governing equations have been transformed into a set of ordinary differential equations, which are nonlinear and cannot be solved analytically, therefore, fourth order Runge-Kutta method along with shooting technique has been used for solving it.

The results for velocity, microrotation, temperature and concentration functions are carried out for the wide range of important parameters namely, material parameter, magnetic parameter, unsteadiness parameter, heat source/sink and chemical reaction parameter. The skin friction, the rate of heat transfer and the rate of mass transfer have also been computed.

\section{Mathematical Formulation}

Consider an unsteady, two-dimensional laminar flow of an incompressible micropolar fluid over a stretching sheet. At time $t=0$, the sheet is impulsively stretched with velocity $U_{w}(x, t)$ along the $x$-axis, keeping the origin fixed in the fluid of ambient temperature $T_{\infty}$ and ambient temperature $C_{\infty}$. The stationary Cartesian coordinate system has its origin located at the leading edge of the sheet with the positive $x$-axis extending along the sheet, while the $y$-axis is measured normal to the surface of the sheet.

A schematic representation of the physical model and coordinates system is depicted in Fig. A. A uniform magnetic field of strength $\mathrm{B}_{0}$ is assumed to be applied in the positive $y$-direction normal to the plate. The magnetic Reynolds number of the flow is taken to be small enough so that the induced magnetic field is negligible. Hall effects and Joule heating are negligible.

The level of concentration of foreign mass is assumed to be low, so that the Soret and Dufour effects are negligible. Under these assumptions along with the Boussinesq and boundary layer approximations, the system of equations, which models the flow is given by

Continuity equation

$\frac{\partial u}{\partial x}+\frac{\partial v}{\partial y}=0$

Linear momentum equation

$\frac{\partial u}{\partial t}+u \frac{\partial u}{\partial x}+v \frac{\partial u}{\partial y}=\left(\frac{\mu+\kappa}{\rho}\right) \frac{\partial^{2} u}{\partial y^{2}}-\frac{\sigma B_{0}^{2}}{\rho} u+\frac{\kappa}{\rho} \frac{\partial N}{\partial y}$ 
Angular momentum equation

$\rho j\left(\frac{\partial N}{\partial t}+u \frac{\partial N}{\partial x}+v \frac{\partial N}{\partial y}\right)=\gamma \frac{\partial^{2} N}{\partial y^{2}}-\kappa\left(2 N+\frac{\partial u}{\partial y}\right)$

Energy equation

$\frac{\partial T}{\partial t}+u \frac{\partial T}{\partial x}+v \frac{\partial T}{\partial y}=\alpha \frac{\partial^{2} T}{\partial y^{2}}+q\left(T-T_{\infty}\right)$

Species equation

$\frac{\partial C}{\partial t}+u \frac{\partial C}{\partial x}+v \frac{\partial C}{\partial y}=D \frac{\partial^{2} C}{\partial y^{2}}-k_{0}\left(C-C_{\infty}\right)$

The boundary conditions for the velocity, temperature and concentration fields are

$$
\begin{gathered}
u=U_{w}, v=0, N=-m \frac{\partial u}{\partial y}, \frac{\partial T}{\partial y}=-\frac{q_{w}}{k}, \frac{\partial C}{\partial y}=-\frac{M_{n}}{D} \\
\text { at } \quad y=0
\end{gathered}
$$$$
u \rightarrow 0, N \rightarrow 0, T \rightarrow T_{\infty}, C \rightarrow C_{\infty}
$$$$
y \rightarrow \infty
$$

where $m$ is the boundary parameter with 0 $\leq m \leq 1 \quad$ (21), $u$ and $v$ are the velocity components in the $x$ - and $y$-directions, respectively, $T$ is the fluid temperature in the boundary layer, $C$ is the fluid temperature in the boundary layer, $N$ is the microrotation or angular velocity, and $j, \gamma, \mu, \kappa, \rho$, and $\alpha$ are the microinertia per unit mass, spin gradient viscosity, dynamic viscosity, vortex viscosity, fluid density and thermal diffusivity, respectively. It is assumed that the stretching velocity $U_{w}(x, t)$, the surface heat flux $q_{w}(x, t)$ and the surface mass flux $M_{n}(x, t)$ are of the forms

$$
U_{w}(x, t)=\frac{a x}{1-d t}, q_{w}(x, t)=\frac{b x}{1-d t}, M_{n}(x, t)=\frac{c x}{1-d t}
$$

where $a, b, c$ and $d$ are constants with $a>0, b \geq 0, c \geq 0$ and $d \geq 0$ (with $d t<1$ ), and both $a$ and $d$ have dimension time ${ }^{-1}$. It should be noted that at $t=0$ (initial motion), equations. (2.1) - (2.4) describe the steady flow over a stretching surface. These particular forms of $U_{w}(x, t), q_{w}(x, t)$ and $M_{n}(x, t)$ have been chosen in order to be able to devise a new similarity transformation, which transforms the governing partial differential equations (2.1) - (2.4) into a set of ordinary differential equations, thereby facilitating the exploration of the effects of the controlling parameters (see Andersson et al. (22)).

As was shown by Ahmadi (21), the spingradient viscosity $\gamma$ can be defined as

$$
\gamma=(\mu+\kappa / 2) j=\mu(1+K / 2) j
$$

where $K=\kappa / \mu$ is the dimensionless viscosity ratio and is called the material parameter.

The continuity equation (2.1) is satisfied by the Cauchy Riemann equations

$u=\frac{\partial \psi}{\partial y}$ and $v=-\frac{\partial \psi}{\partial x}$

where $\psi(x, y)$ is the stream function.

In order to transform equations (2.2) to (2.6) into a set of ordinary differential equations, the following similarity transformations and dimensionless variables are introduced. 


$$
\begin{aligned}
& f(\eta)=\frac{\psi}{\left(v x U_{w}\right)^{1 / 2}}, \quad \eta=y \sqrt{\frac{U_{w}}{v x}}, \\
& N=U_{w}\left(\frac{U_{w}}{v x}\right)^{1 / 2} g(\eta), \\
& \theta(\eta)=\frac{k\left(T-T_{\infty}\right)}{q_{w}}\left(\frac{U_{w}}{v x}\right)^{1 / 2} \\
& , \phi(\eta)=\frac{k\left(C-C_{\infty}\right)}{M_{n}}\left(\frac{U_{w}}{v x}\right)^{1 / 2}, \\
& M=\frac{\sigma B_{0}^{2}}{\rho U_{w}}, S=\frac{d}{a}, \quad Q=\frac{Q_{0} k}{a}, \quad \operatorname{Pr}=\frac{v}{\alpha}, \\
& S c=\frac{v}{D}(2.10)
\end{aligned}
$$

where $f(\eta)$ is the dimensionless stream function, $\theta$ - dimensionless temperature, $\phi$ dimensionless concentration, $\eta$ - similarity variable, $M$ - the Magnetic parameter, $S$ - the unsteadiness parameter, $Q$ - the heat source/sink parameter, $P r$ - the Prandtl number and $K r$-the chemical reaction parameter.

In view of equations (2.9) and (2.10), the equations (2.2) to (2.6) transform into

$(1+K) f^{\prime \prime \prime}+f f^{\prime \prime}-f^{\prime 2}+K g^{\prime}-M f^{\prime}-S\left(f^{\prime}+\frac{1}{2} \eta f^{\prime \prime}\right)=0$

$\left(1+\frac{K}{2}\right) g^{\prime \prime}+f g^{\prime}-f^{\prime} g-K\left(2 g+f^{\prime \prime}\right)-S\left(\frac{3}{2} g+\frac{1}{2} \eta g^{\prime}\right)=0$

$\frac{1}{\operatorname{Pr}} \theta^{\prime \prime}+f \theta^{\prime}-f^{\prime} \theta+Q \theta-S\left(\theta+\frac{1}{2} \eta \theta^{\prime}\right)=0$

$\frac{1}{S c} \phi^{\prime \prime}+f \phi^{\prime}-f^{\prime} \phi-K r \phi=0$
The corresponding boundary conditions are:

$$
\begin{aligned}
& f(0)=0, f^{\prime}(0)=0, g=-m f^{\prime \prime}(0), \theta^{\prime}(0)=-1, \phi^{\prime}(0)=-1 \\
& f^{\prime} \rightarrow 0, g \rightarrow 0, \theta \rightarrow 0, \phi \rightarrow 0 \\
& \text { as } \quad \eta \rightarrow \infty
\end{aligned}
$$

where the primes denote differentiation with respect to $\eta$

The physical quantities of interest are the skin friction coefficient $C_{f}$, the local Nusselt

number $N u$ and Sherwood number $S h$ which are defined as

$$
\begin{aligned}
C_{f} & =\frac{\tau_{w}}{\rho U_{w}{ }^{2} / 2}, \\
S h & =\frac{x M_{n}}{k\left(C_{w}-C_{\infty}\right)}
\end{aligned}
$$

where the wall shear stress $\tau_{w}$, the heat flux $q_{w}$ and mass flux $M_{n}$ are given by

$$
\begin{aligned}
& \tau_{w}=\left[(\mu+\kappa) \frac{\partial u}{\partial y}+\kappa N\right]_{y=0}, \\
& q_{w}=-k\left(\frac{\partial T}{\partial y}\right)_{y=0} M_{n}=-k\left(\frac{\partial C}{\partial y}\right)_{y=0}
\end{aligned}
$$

Thus, we get

$$
\begin{aligned}
& \frac{1}{2} C_{f} \operatorname{Re}_{x}=\left(1+\frac{K}{2}\right) f^{\prime \prime}(0), \\
& N u / \operatorname{Re}_{x}^{1 / 2}=1 / \theta(0), S h / \operatorname{Re}_{x}^{1 / 2}=1 / \phi(0)
\end{aligned}
$$

Our main aim is to investigate how the values of $f^{\prime \prime}(0), 1 / \theta(0)$ and $1 / \phi(0)$ vary in terms of the governing parameters. 


\section{Solution of the Problem}

The set of coupled non-linear governing boundary layer equations (2.11) - (2.14) together with the boundary conditions (2.15) are solved numerically by using RungeKutta fourth order technique along with shooting method. First of all, higher order non-linear differential Equations (2.11) (2.14) are converted into simultaneous linear differential equations of first order and they are further transformed into initial value problem by applying the shooting technique (Jain et al.(23)). The resultant initial value problem is solved by employing RungeKutta fourth order technique. The step size $\Delta \eta=0.05$ is used to obtain the numerical solution with five decimal place accuracy as the criterion of convergence. From the process of numerical computation, the skinfriction coefficient, the Nusselt number and the Sherwood number, which are respectively proportional to $f^{\prime \prime}(0), 1 / \theta(0)$ and $1 / \phi(0)$, are also sorted out and their numerical values are presented in a tabular form.

\section{Results and Discussion}

The governing equations (2.11) - (2.14) subject to the boundary conditions (2.15) are integrated as described in section 3 . In order to get a clear insight of the physical problem, the velocity, temperature and concentration have been discussed by assigning numerical values to the parameters encountered in the problem. The effects of various parameters on velocity profiles in the boundary layer are depicted in Figs. 1-4. The effects of material parameter on Angular velocity profiles in the boundary layer are depicted in Figs.5-8. The effects of various parameters on temperature profiles in the boundary layer are showen in Figs. 914. The effects of various parameters on concentration profiles in the boundary layer are depicted in Figs. 15-20.

Fig. 1 shows the dimensionless velocity profiles for different values of magnetic parameter $(M)$. It is seen that, as expected, the velocity decreases with an increase of magnetic parameter. The magnetic parameter is found to retard the velocity at all points of the flow field. It is because that the application of transverse magnetic field will result in a resistive type force (Lorentz force) similar to drag force which tends to resist the fluid flow and thus reducing its velocity. Also, the boundary layer thickness decreases with an increase in the magnetic parameter. Fig.2 illustrates the effect of the unsteadiness parameter $(S)$ on the velocity field. It is observed that as the unsteadiness parameter increases, the velocity field decreases. The effect of material parameter $(K)$ on the velocity is illustrated in Fig.3. It is noticed that the velocity increases with increasing values of the material parameter.

Fig. 4 shows the variation of the velocity with the boundary parameter $(m)$. It is noticed that the velocity decreases with an increase in the boundary parameter.

Fig.5 illustrates the effect of magnetic parameter on the angular velocity. It is noticed that as the magnetic parameter increases, the angular velocity increases. Fig. 6 shows the variation of the angular velocity with the unsteadiness parameter. It is noticed that the angular velocity thickness increases near the sheet $(\eta \leq 1)$ and decreases for $(\eta>1)$ with an increase in the unsteadiness parameter. Fig. 7 depicts the angular velocity with the material parameter. It is noticed that the angular velocity decreases with an increase in the material parameter. The effect of the boundary parameter on the angular velocity is illustrated in Fig. 8. It is observed that as the boundary parameter increases, the angular 
velocity increases. The effect of the magnetic parameter on the temperature is illustrated in Fig.9. It is observed that as the magnetic parameter increases, the temperature increases. Fig. 10 depicts the thermal boundary-layer with the unsteadiness parameter. It is noticed that the thermal boundary layer thickness decreases with an increase in the unsteadiness parameter.

Fig. 11 depicts the thermal boundary-layer with the material parameter. It is noticed that the thermal boundary layer thickness decreases with an increase in the material parameter.
Fig.12 illustrates the effect of the boundary parameter on the temperature. It is noticed that as the boundary parameter increases, the temperature increases. Fig. 13 shows the variation of the thermal boundary-layer with the Prandtl number $(\mathrm{Pr})$. It is noticed that the thermal boundary layer thickness decreases with an increase in the Prandtl number. Fig. 14 shows the variation of the thermal boundary-layer with the heat source/sink parameter $(Q)$. It is observed that the thermal boundary layer thickness increases with an increase in the heat source/sink.

Table.1 Comparison of for Different Values of $\mathrm{S}$ and $\operatorname{Pr}$ when $\mathrm{K}=\mathrm{Q}=\mathrm{Sc}=\mathrm{Kr}=\mathrm{m}=0$

\begin{tabular}{|c|c|c|c|c|}
\hline$S$ & $P r$ & \multicolumn{3}{|c|}{$\theta(0)$} \\
\cline { 3 - 5 } & & Elbashbeshy(5) & Bachok et al. (6) & Present Study \\
\hline 0 & 0.72 & 1.2253 & 1.2367 & 1.2258 \\
0 & 1 & 1.0000 & 1.0000 & 1.0000 \\
1 & 0.72 & & 0.9116 & 0.9116 \\
1 & 1 & & 0.8591 & 0.8591 \\
\hline
\end{tabular}

Fig.A Schematic Representation of the Physical Model and Coordinate System

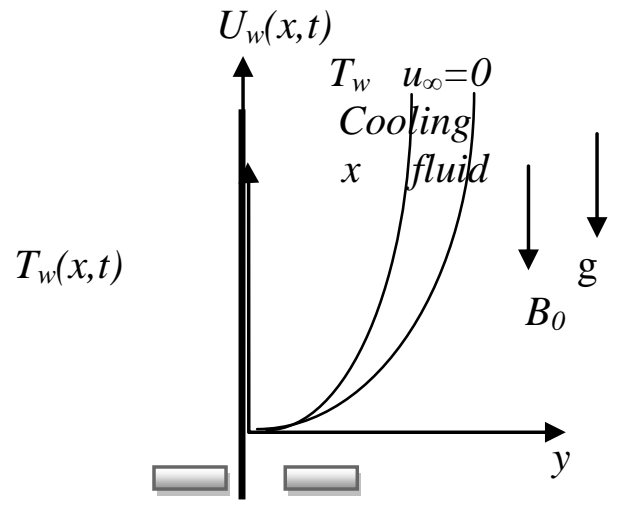

Slot 
Fig.1 Velocity Profiles for Different Values of M

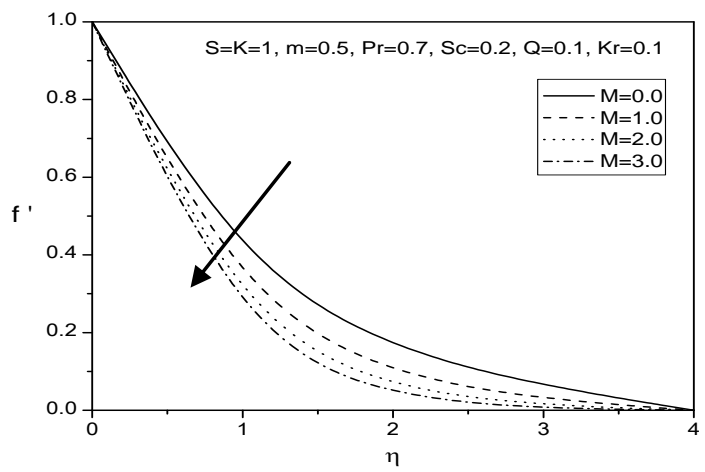

Fig.2 Velocity Profiles for Different Values of S

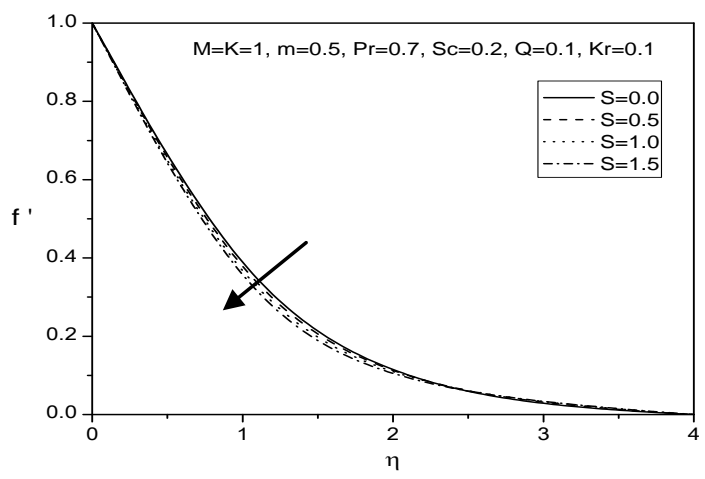

Fig.3 Velocity Profiles for Different Values of K

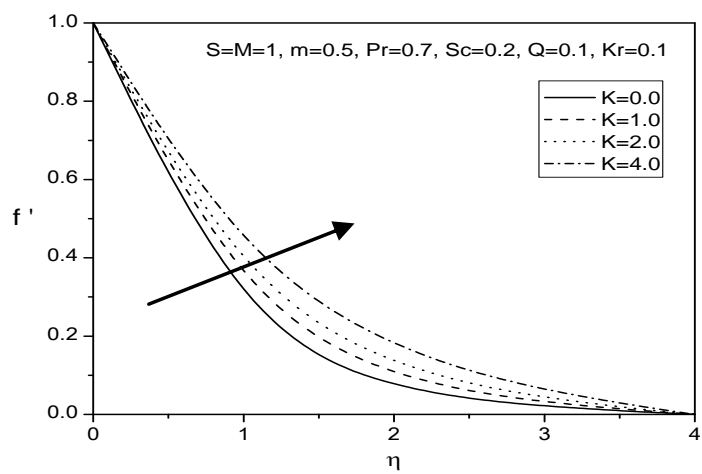


Fig.4 Velocity Profiles for Different Values of m

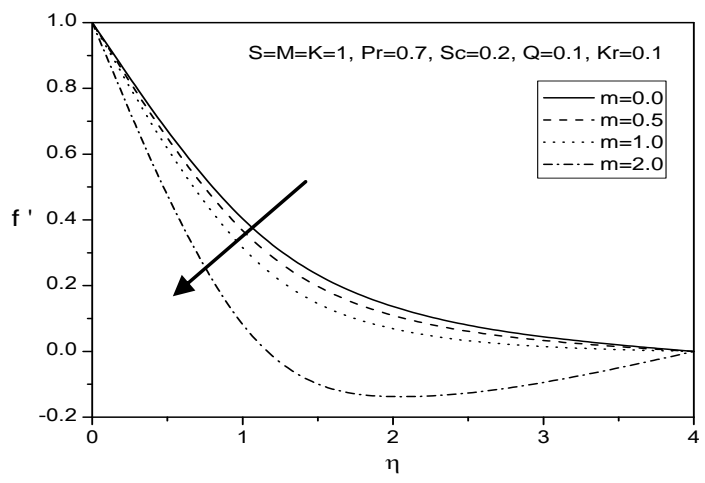

Fig.5 Angular velocity Profiles for Different Values of M

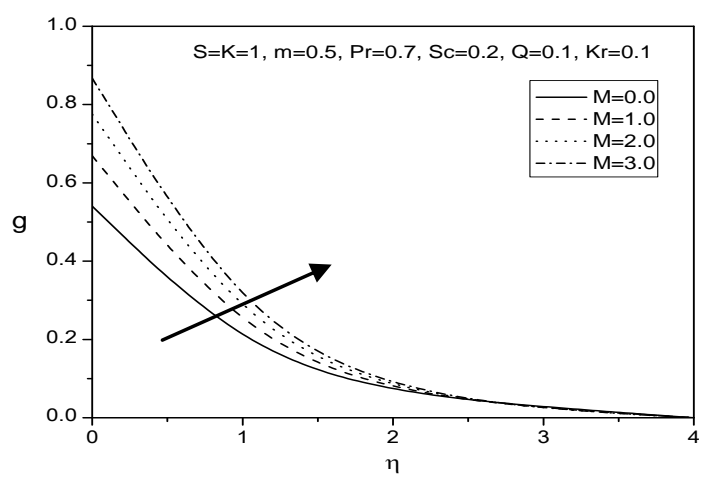

Fig.6 Angular Velocity Profiles for Different Values of S

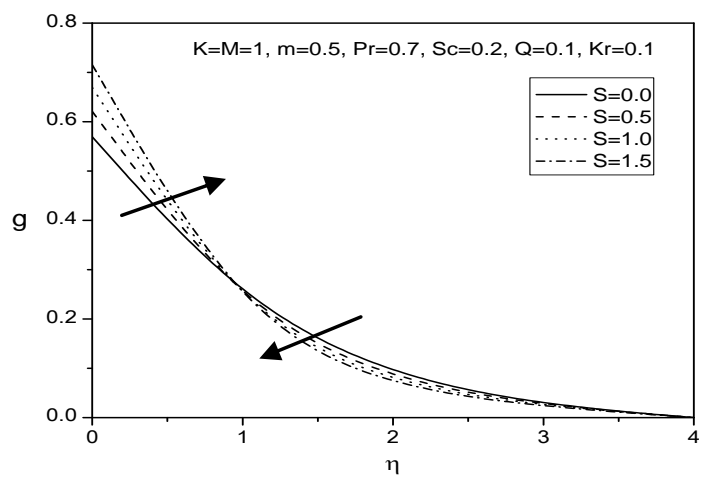


Fig.7 Angular Velocity for Different Values of K

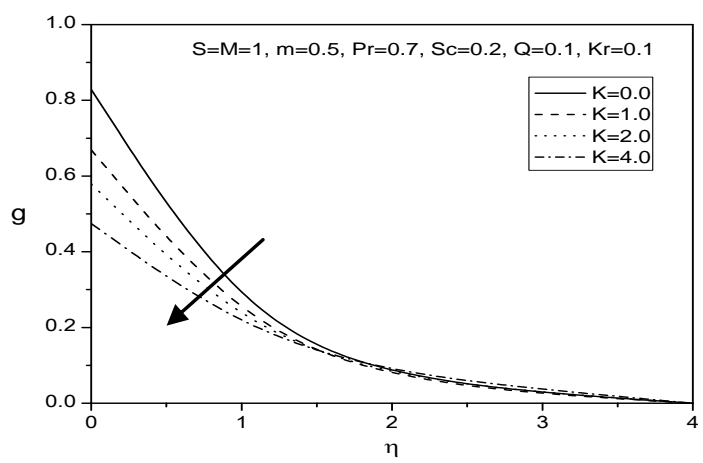

Fig.8 Angular Velocity for Different Values of m

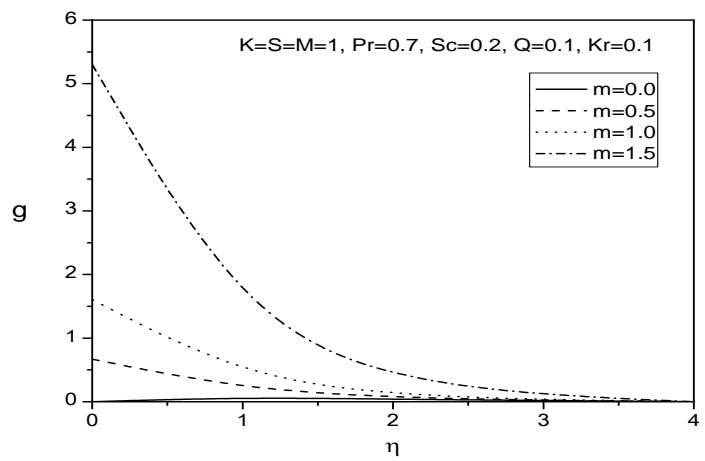

Fig.9 Temperature for Different Values of M

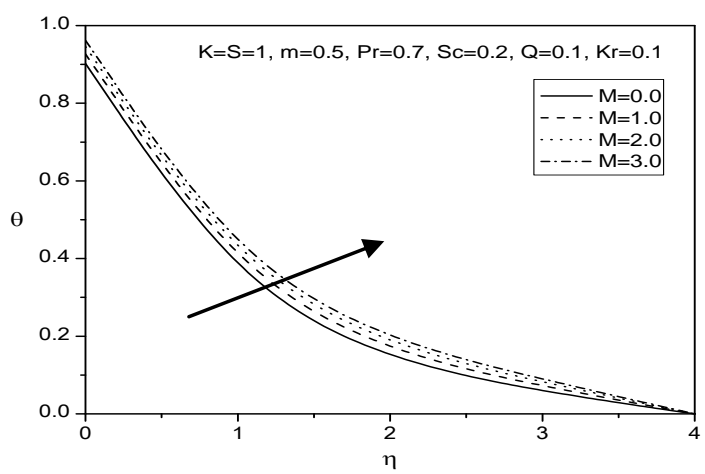


Fig.10 Temperature for Different Values of S

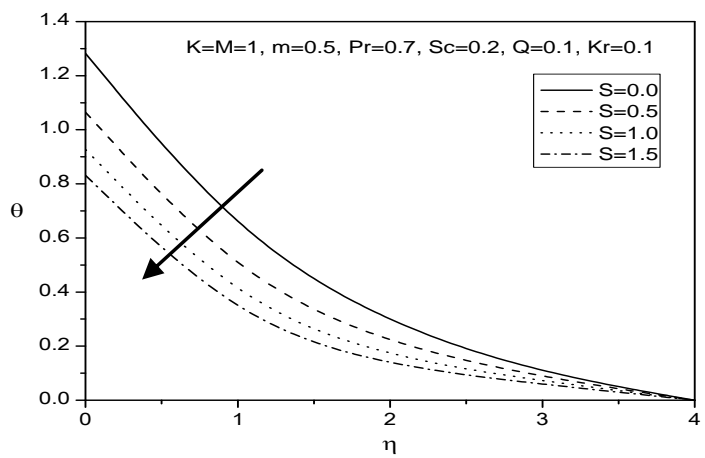

Fig.11 Temperature for Different Values of K

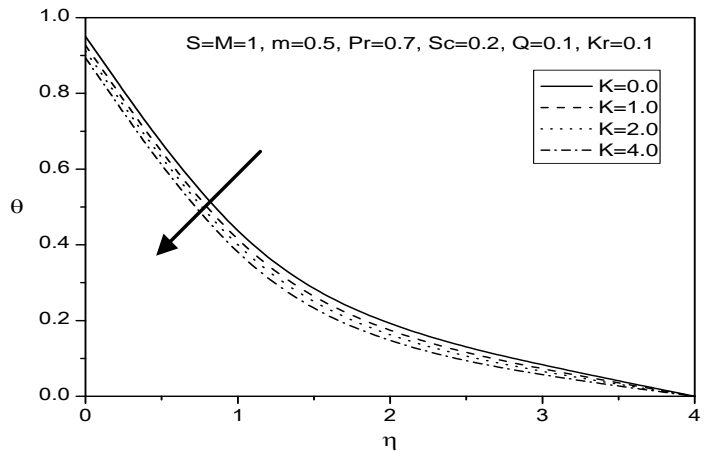

Fig.12 Temperature for Different Values of m

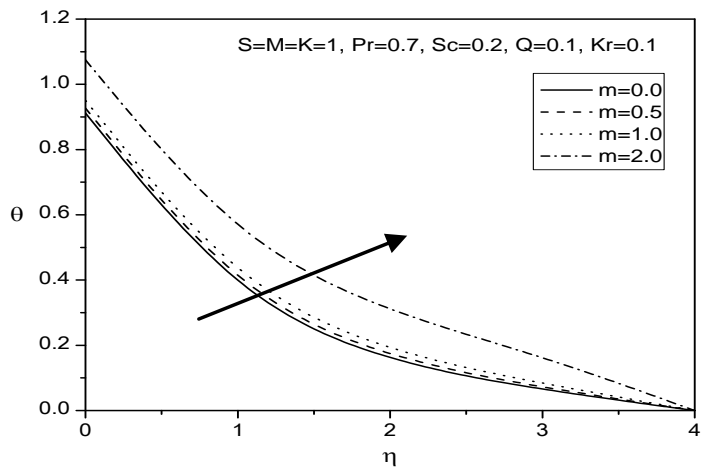


Fig.13 Temperature for Different Values of $\operatorname{Pr}$

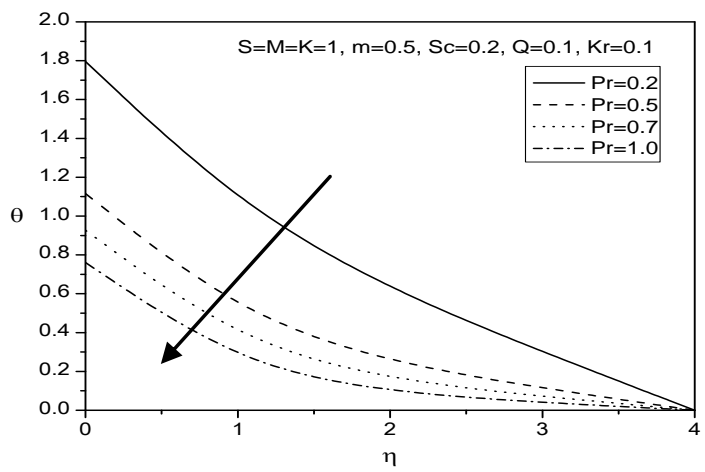

Fig.14 Temperature for Different Values of Q

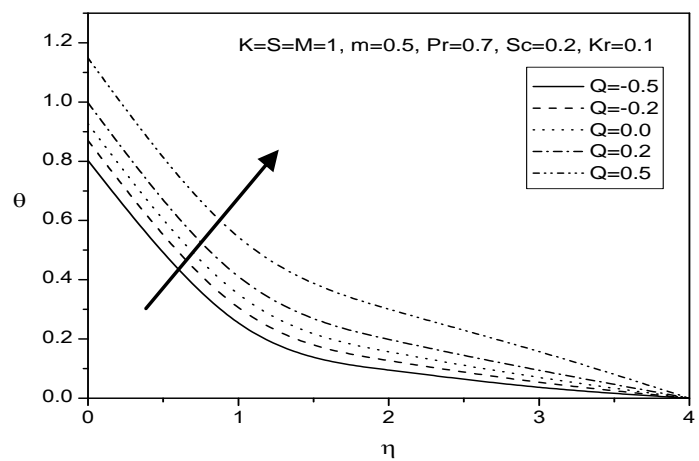

Fig.15 Concentration for Different Values of M

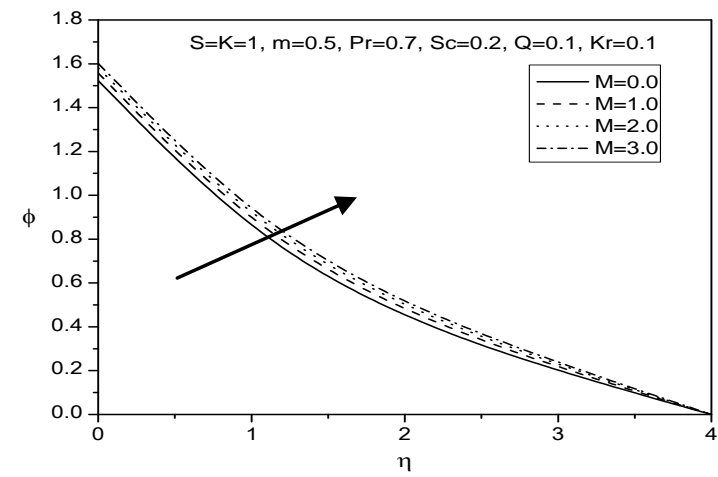


Fig.16 Concentration for Different Values of S

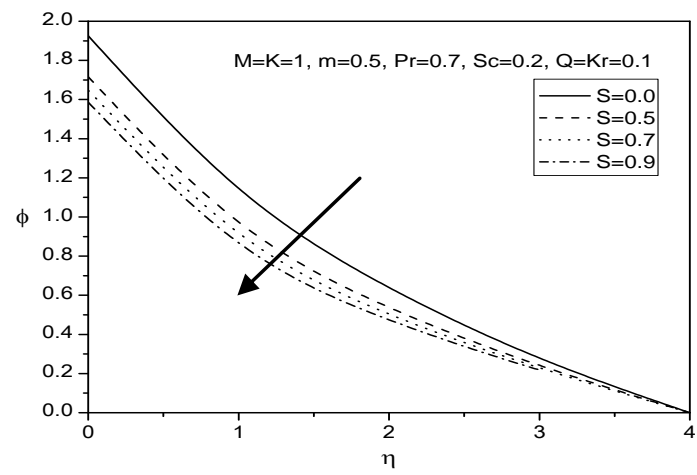

Fig.17 Concentration for Different Values of K

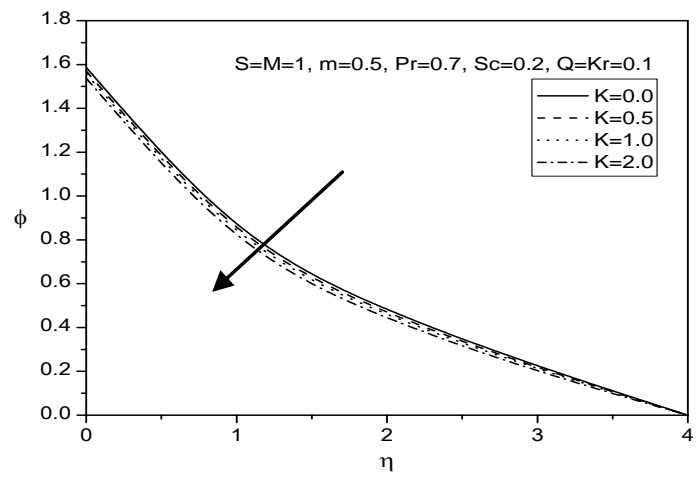

Fig.18 Concentration for Different Values of m

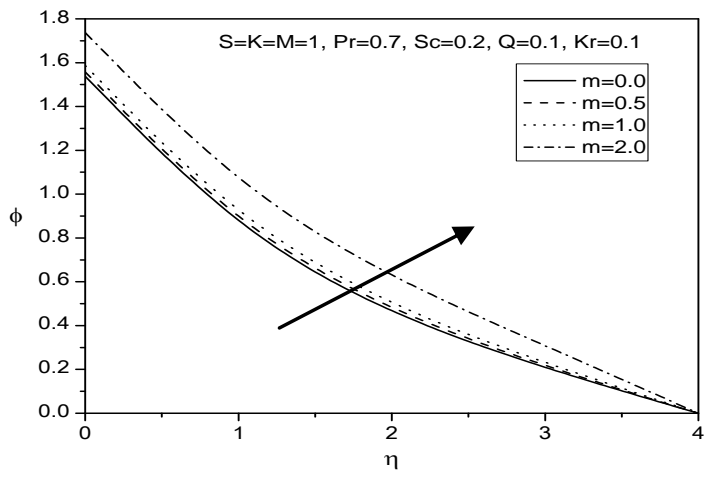


Fig.19 Concentration for Different Values of Sc

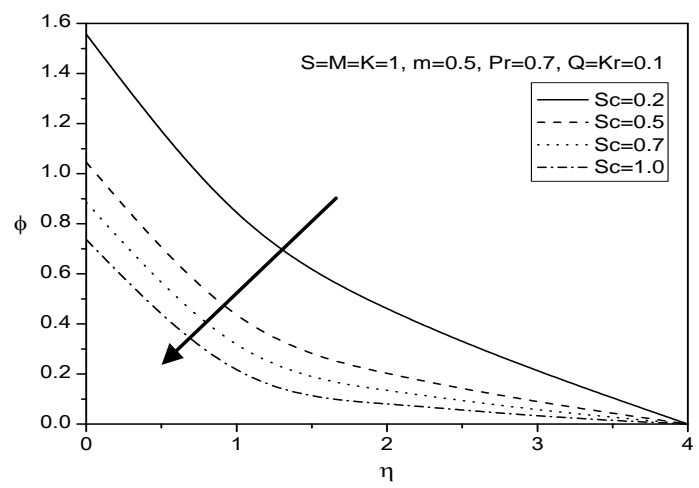

Fig.20 Concentration for Different Values of $\mathrm{Kr}$

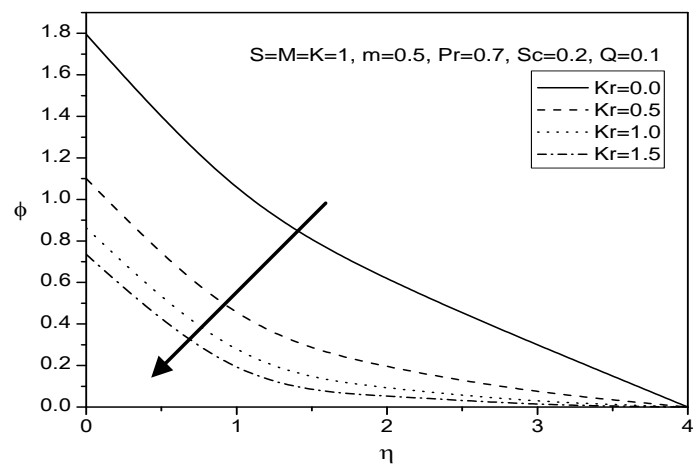

Fig.21 Variation of the Skin-friction with $\mathrm{K}$ for Different $\mathrm{M}$

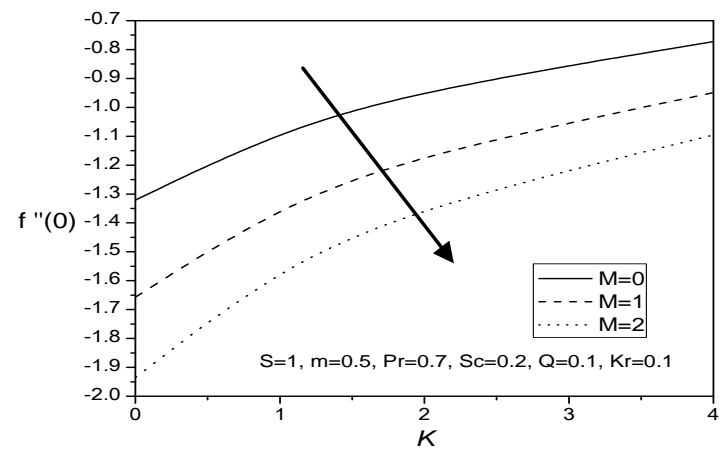


Fig.22 Variation of the Nusselt Number with K for Different Q

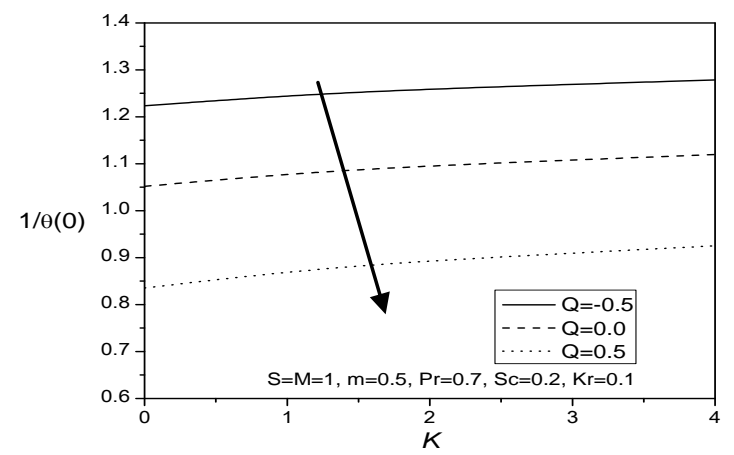

Fig.23 Variation of the Sherwood Number with K for Different Kr

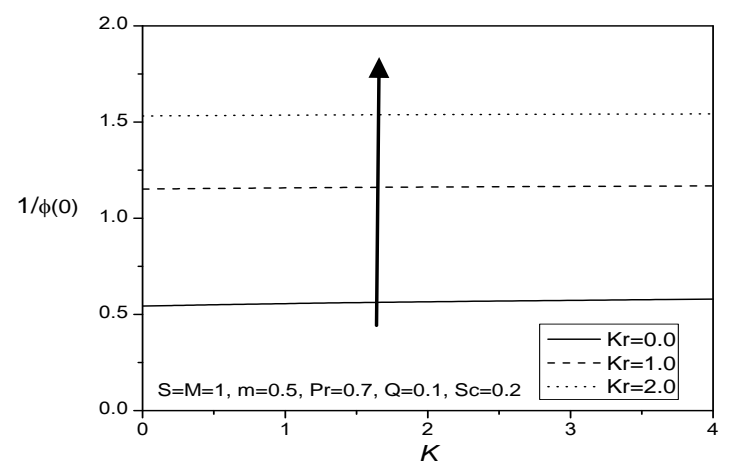

The effect of magnetic parameter on the concentration field is illustrated Fig.15. As the magnetic parameter increases the concentration is found to be increasing. The effect of unsteadiness parameter on the concentration field is illustrated Fig. 16. It is noticed that the concentration boundary layer thickness decreases with an increase in the unsteadiness parameter. The effect of material parameter on the concentration field is illustrated Fig. 17. It is noticed that the concentration boundary layer thickness decreases with an increase in the material parameter. Fig. 18 illustrates the effect of boundary parameter on the concentration. As the boundary parameter increases, an increasing trend in the concentration field is noticed. The influence of the Schmidt number $(S c)$ on the concentration field is shown in Fig.19. It is noticed that the concentration decreases with the increase of the Schmidt number. The influence of the chemical reaction parameter $(K r)$ on the concentration field is shown in Fig.20. It is noticed that the concentration decreases with the increase of the chemical reaction parameter.

Fig. 21 shows the variation of the skin friction with for different values of magnetic parameter. It is observed that the skin friction decreases with an increase in the magnetic parameter. Fig. 22 depicts the variation of the heat transfer rate with for different values of heat source/sink parameter. It is noticed that the heat transfer rate decreases with an increase in the heat source/sink parameter. Fig. 23 shows the variation of the mass transfer rate with for different values of chemical reaction parameter. It is observed that the mass transfer rate increases with an increase in the chemical reaction parameter.

For validation of the numerical method used in this study, results for $\theta(0)$, were 
compared with those of Elbabeshy (5) and Blocks et al. (6) for various values of $S$ and $P r$. The quantitative comparison is shown in Table 1 and it is found to be in excellent agreement.

\section{Conclusion}

In the present chapter, an unsteady magnetohydrodynamic (MHD) laminar flow past a stretching sheet with prescribed surface heat and mass flux by taking mass transfer, heat source or sink and chemical reaction effects into account, is analyzed. The governing equations are approximated to a system of non-linear ordinary differential equations by similarity transformation. Numerical calculations are carried out for various values of the dimensionless parameters of the problem. It has been found that

1. The velocity decreases while the angular velocity, temperature and concentration functions increase with an increase in the magnetic parameter.

2. The velocity increases while the angular velocity, temperature and concentration functions decrease with an increase in the material parameter.

3. The heat source/sink enhances the temperature.

4. The chemical reaction reduces the concentration.

5. The skin friction reduces as the magnetic parameter increases and the increases as the material parameter increases.

6. The heat source/sink reduces the heat transfer rate.

7. The chemical reaction or material parameter enhances the mass transfer rate.

\section{References}

1. Eringen, A. C., (1966), Theory of micropolar fluids, Journal of Mathematics and Mechanics, Vol.16, pp. 1-18.
2. Na, T. Y. and Pop, I., (1997), Boundarylayer flow of micropolar fluid due to a stretching wall, Archives of Applied Mechanics, Vol.67, No.4, pp. 229-236.

3. Desseaux, A. and Kelson, N. A., (2000), Flow of a micropolar fluid bounded by a stretching sheet, Anziam J., Vol.42, pp.536-560.

4. Takhar, H. S., Agarwal, R. S., Bhargava, R., and Jain, S., (1998), Mixed convection flow of a micropolar fluid over a stretching sheet, Heat and Mass Transfer, Vol.34, pp.213-219.

5. Elbashbeshy, E. M. A., (1998), Heat transfer over a stretching surface with variable surface heat flux, J. Phys. D: Appl. Phys., Vol. 31, pp. 1951-1954.

6. Bachok, N., Ishak, A., and Nazar, R., (2010), Flow and heat transfer over an unsteady stretching sheet in a micropolar fluid with preseribed surface heat flux, International journal of mathematical models and methods in applied sciences, Issue 3, Vol. 4, pp.167-176.

. Vajravelu, K., and Rollins, D., (1992), Heat transfer in electrically conducting fluid over a stretching sheet, Int J Nonlinear Mech, Vol.27, No.2, pp.265-277.

8. Prasad, K.V., Vajravelu, K., and Datti, P.S., (2010), The effects of variable fluid properties on the hydro-magnetic flow and heat transfer over a non-linearly stretching sheet, Int J Thermal Sciences, Vol.49, pp.603-610.

9. Haque, Md. Z., Alam, Md. M., Ferdows, M., Postelnicu, A., (2011), Micropolar fluid behaviors on steady MHD free convection flow and mass transfer with constant heat and mass fluxes, joule heating and viscous dissipation. J.King\ Saud Univ. Engg.Sci doi:10.1016/j.jksues.2011.02.003, 2011.

10. Rehbi, A.D., Tariq, A.A., Benbella, A.S., and Mahoud, A.A., (2007), Unsteady natural convection heat transfer of micropolar fluid over a vertical surface with constant Heat flux, Turkish J. Eng. Env. Sci., Vol. 31, pp. 225-233. 
11. Olajuwon, B.I., and Oahimire, J.I., (2013), unsteady free convection heat and mass transfer in an mhd micropolar fluid in the presence of thermo diffusion and thermal radiation, International Journal of Pure and Applied Mathematics, Vol. 84 No. 2, pp. 15-37.

12. Cortell, R., (2005), Flow and heat transfer of a fluid through a porous medium over a stretching surface with internal heat generation/ absorption and suction/blowing, Fluid Dynam. Res., Vol.37, pp.231-245.

13. Mohamad, (2009), Double diffusive convection-radiation interaction on unsteady MHD flow over a vertical moving porous plate with heat generation and soret effect, Applied mathematical sciences, Vol13, pp. 629-651.

14. Mostafa, A.A., Mahmoud, Shimaa, E., (2012), MHD flow and heat transfer of a micropolar fluid over a stretching surface with heat generation (absorption) and slip velocity, Waheed Journal of the Egyptian Mathematical Society, Vol20, pp.20-27

15. Khedr, M. E., Chamkha, A. J., and Bayomi, M., MHD flow of a micropolar fluid past a stretched permeable surface with heat generation or absorption. Nonlinear analysis modeling and control, Vol. 14, No 1,pp.27-40

16. Abo-Eldahab, E.M., and Abd El-Aziz, M., (2005), Hydromagnetic three-dimensional free convection heat transfer over a stretch- ing surface embedded in a nonDarcian porous medium in the presence of heat generation or absorption, Candian $\mathbf{J}$ Physics, Vol.83, pp.739-751.
17. Damseh, R.A., Al Odat, M.Q., Chamkha, A.J., and Shannak, B.A., (2009), Combined effect of heat generation or absorption and first-order chemical reaction on micropolar fluid flows over a uniformly stretched permeable surface, Int. J. Therm. Sci., Vol.48, pp.1658-1663.

18. Kandasamy, R., Periasamy, K., and Sivagnana, K., (2005), Chemical reaction, heat and mass transfer on MHD flow over a vertical stretching surface with heat source and stratification effects, Int. J. of heat and mass transfer, Vol.48, pp.45-57.

19. Seddek, M.A., (2005), Finite-elêment method for effects of chemical reaction, variable viscosity, thermophoresis and heat generation/absorption on a boundary layer hydromagnetic flow with heat and mass transfer over a heat surface, Acta mech., Vol.177,pp. 1-18.

20. Ishak, A., Nazar, R., and Pop, I., (2008), Mixed convection stagnation point flow of a micropolar fluid towards a stretching sheet, Meccanica, Vol. 43, pp. 411-418.

21. Ahmadi, G., (1976), Self-similar solution of incompressible micropolar boundary layer flow over a semi-infinite plate, Int. J. Eng. Sci., Vol. 14, pp. 639-646.

22. Andersson, H. I., Aarseth, J. B., and Dandapat, B. S., (2000), Heat transfer in a liquid film on an unsteady stretching surface, Int. J. Heat Mass Transfer, Vol. 43, pp. 69-74.

23. Jain, M.K., Iyengar, S.R.K. and Jain, R.K., (1985), Numerical Methods for Scientific and Engineering Computation, Wiley Eastern Ltd., New Delhi, India.

\section{How to cite this article:}

Sreedhar Babu, M., M. Lavanya and Venkata Ramanaiah, G. 2016. MHD flow of heat and mass transfer over an unsteady stretching sheet in a micropolar fluid with heat source/sink and chemical reaction. Int.J.Curr.Res.Aca.Rev. 4(1): 91-107

doi: http://dx.doi.org/10.20546/ijcrar.2016.401.009 